\title{
Second harmonic generation response by gold nanoparticles at the polarized water/2-octanone interface: from dispersed to aggregated particles
}

\author{
P Galletto ${ }^{1}$, H H Girault ${ }^{1}$, C Gomis-Bas ${ }^{2}$, D J Schiffrin ${ }^{2}$, R Antoine ${ }^{3}$, \\ M Broyer ${ }^{3}$ and P F Brevet ${ }^{3,4}$ \\ ${ }^{1}$ Laboratoire d'Electrochimie Physique et Analytique, Ecole Polytechnique Fédérale de Lausanne, \\ CH-1015 Lausanne, Switzerland \\ 2 Centre for Nanoscale Science, Department of Chemistry, University of Liverpool, Liverpool \\ L69 3BX, UK \\ ${ }^{3}$ Université Lyon 1, Laboratoire de Spectrométrie Ionique et Moléculaire, UMR CNRS 5579, 43 \\ boulevard du 11 Novembre 1918, F-69622 Villeurbanne Cedex, France \\ E-mail: pfbrevet@lasim.univ-lyon1.fr
}

Received 14 December 2006, in final form 22 March 2007

Published 13 August 2007

Online at stacks.iop.org/JPhysCM/19/375108

\begin{abstract}
Gold nanoparticles with a diameter of approximately $20 \mathrm{~nm}$ have been observed at the polarized water/2-octanone interface by the nonlinear optical technique of second harmonic generation. Electric field induced adsorption of the gold particles at this liquid/liquid interface is clearly observed and confirms that these are negatively charged. The process is quasi-reversible at high potential sweep rates, but aggregation at the interface is observed at slower sweep rates through the loss of the nonlinear optical signal. The time evolution of the second harmonic signal is also reported during potential step experiments. After a rapid increase due to adsorption, a continuous decrease in the nonlinear optical signal intensity is observed due to aggregation of the particles into large islands at the interface. Diffusion of these large islands at the interface was observed for a longer timescale through large signal fluctuations.
\end{abstract}

\section{Introduction}

Metal colloids have been investigated extensively in the past due to their potential applications in many fields such as nanoelectronics or bioanalytical science. As a result, a great deal of work has been devoted to their synthesis and a wide range of techniques has been used for their characterization, in particular optical and microscopy techniques [1, 2]. The literature on their properties at interfaces, and more specifically at liquid interfaces, is rather scarce. Electrodeposition of gold at the polarized liquid/liquid interface by two-phase reduction was

4 Author to whom any correspondence should be addressed. 
observed by Cheng et al [3]. The nanoparticles were produced directly at the interface and not in the bulk, and this experiment clearly demonstrated that the externally applied potential across the interface could be used to control nanoparticle synthesis. This work also showed that growth could be monitored in situ by ultraviolet-visible (UV-vis) absorbance spectroscopy, which is a linear optical spectroscopy technique. Further work on two-dimensional films of hydrophobic metallic particles at the air-water interface has also been reported with the intent of investigating the conductivity of the film as a function of the average particle distance. Large enhancements of the nonlinear optical response were briefly discussed [4]. The present work makes use of the nonlinear optical technique of surface second harmonic generation (SHG) as a surface-sensitive method to study gold particles adsorbed at the polarized liquid/liquid interface. This is an important question that relates to the stability of growing clusters under an externally applied interfacial potential, since the electrochemical synthesis route would require the control of the adsorption properties of already grown particles. This extra degree of freedom would be an advantage in studies where particle-particle interactions are critical to the stability of the system studied [5]. Nanoparticle stabilization is achieved by using organic ligands to passivate the particles and hence prevent their coalescence into large ensembles. The problem of the stability of the adsorbed particles can indeed be of great relevance in interfacial studies. For instance, the synthesis of the particles and their subsequent use at the polarized liquid/liquid interface in a catalytic heterogeneous reaction has been reported [6]. Formation of the particles at the liquid/liquid interface under potential control was observed as well as catalytic activity of the film. However, the exact state of the colloidal material remains largely unknown.

Surface SHG is a process whereby two photons at the fundamental frequency are converted into one photon at the harmonic frequency. In the electric dipole approximation, this process is forbidden in media with inversion symmetry. As a result, this process cannot occur in the bulk of the two immiscible liquid phases forming the liquid/liquid interface but becomes allowed at the interface. The surface specificity and sensitivity of the technique has been used in the past for many different interfaces and is used here to unambiguously attribute a surface signature to the processes monitored [7, 8]. Surface SHG has been used previously to monitor gold colloids at the air-toluene interface, and it has been shown that the origin of the second harmonic signal can be described well by the contribution from the conduction band electrons only, without the interplay of interband transitions. Enhancement of the SHG intensity was also clearly observed when the harmonic frequency was resonant with the surface plasmon (SP) frequency of the particles [9]. This work underlined the adequacy of the use of the SHG technique for metallic nanoparticles present at the interface between two centrosymmetric media. More recently, it has been demonstrated that the origin of the SHG process in gold and silver metallic particles is electric-dipole allowed due to the breaking of symmetry at the particle surface and the deviation of the shape of the particles from perfect spheres [10, 11]. As a consequence, the origin of the second harmonic response for these small particles is not due to the quadrupole moment, as expected for perfect nanospheres, but rather it is of a dipolar nature [12, 13]. This conclusion was obtained from hyper Rayleigh scattering experiments performed in an homogeneous liquid phase. At liquid/liquid interfaces, the interface supporting the particles also introduces an environmental asymmetry in the medium in which the particles are present. It is thus still unknown whether the dominant contribution to the SHG signal arises from the particles themselves or from a polarization induced by the interaction with the interface.

This work therefore aims to address several of these questions. A polarization analysis of the SH intensity is first discussed in order to answer the question of the origin of the response from the liquid/liquid interface in the presence of adsorbed gold nanoparticles. Next, the reversibility of the potential induced adsorption of the particles is discussed and finally the stability of the adsorbed particles is investigated. 


$$
A g|A g C l| \begin{aligned}
& \text { Aqueous } \\
& \text { Gold Colloids } \|
\end{aligned}\left|\begin{array}{c}
\text { mM THATPBCl } \\
(2-\text { octanone })
\end{array}\right| T P B C l A g \mid A g
$$

Figure 1. Electrochemical cell used for the surface SHG experiments.

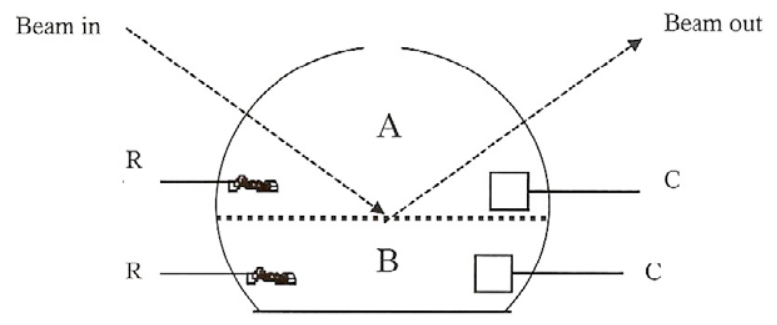

Figure 2. Schematics of the electrochemical cell: (R) reference electrodes; (C) counter electrodes; (A) 2-octanone; (B) aqueous.

\section{Experimental details}

The electrochemical cell used is described in figure 1. The cell that is employed is shown in figure 2. It consisted of a glass cylinder fitted with two Luggin capillaries for the reference electrodes and two platinum wires for the two counter-electrodes. The reference electrode for the aqueous phase was $\mathrm{Ag} / \mathrm{AgCl}$ and for the organic phase it was $\mathrm{Ag} / \mathrm{AgTPBCl}$, prepared according to methods in the literature [14]. All the reported potentials has been transformed into the absolute Galvani potential scale with respect to the organic phase, using the ion transfer potential of tripropylammonium $\left(\mathrm{TPrA}^{+}\right)$as a reference. The organic solvent was 2-octanone and the organic supporting electrolyte was $5 \mathrm{mM}$ tetrahexylammonium tetrakis(4-chlorophenyl) borate (THATPBCl). THATPBCl was prepared by precipitation from an equimolar solution of tetrahexylammonium chloride (THACl) and potassium tetrakis-(4chlorophenyl) borate (KTPBCl) in a 1:3 mixture of water and methanol. After filtering, the precipitate was re-crystallized in acetone. The aqueous phase was a colloidal gold solution prepared according to the method of Turkevich et al [15] by adding $5 \mathrm{ml}$ of a $1 \%$ dihydrate sodium citrate solution to $95 \mathrm{ml}$ of an aqueous solution of hydrogen tetrachloroaurate containing $5 \mathrm{mg}$ of gold and heating under reflux. The colloidal solution had a deep red colour, which is indicative of a gold colloidal suspension with particle diameters of approximately $20 \mathrm{~nm}$. The UV-visible absorption spectrum of the solution (figure 3) exhibited the strong surface plasmon (SP) resonance band at $520 \mathrm{~nm}$ that is characteristic of colloidal gold. No supporting electrolyte was added, since it is known that increasing the salt concentration destabilizes this type of colloidal solution. When used as the aqueous phase in a liquid/liquid system, an appropriate polarization potential window of approximately $200 \mathrm{mV}$ was observed, as shown in figure 4.

The SHG measurements were performed with the fundamental radiation at a wavelength of $1064 \mathrm{~nm}$ from a picosecond Nd-YAG laser delivering pulses of 35 ps duration at a repetition rate of $20 \mathrm{~Hz}$. The average energy per pulse was $4 \mathrm{~mJ}$ and the beam diameter was approximately $4 \mathrm{~mm}$. The laser beam impinged the liquid/liquid interface from the transparent 2-octanone phase and the experiment was hence performed in the total internal reflection (TIR) geometry with an angle of incidence of approximately $72^{\circ}$. The refractive indices of 2-octanone and water in the visible spectrum are 1.416 and 1.333 , respectively, which leads to a critical angle for total internal reflection of $70^{\circ}$. The surface plasmon enhanced SH signal from the interface at $532 \mathrm{~nm}$ 


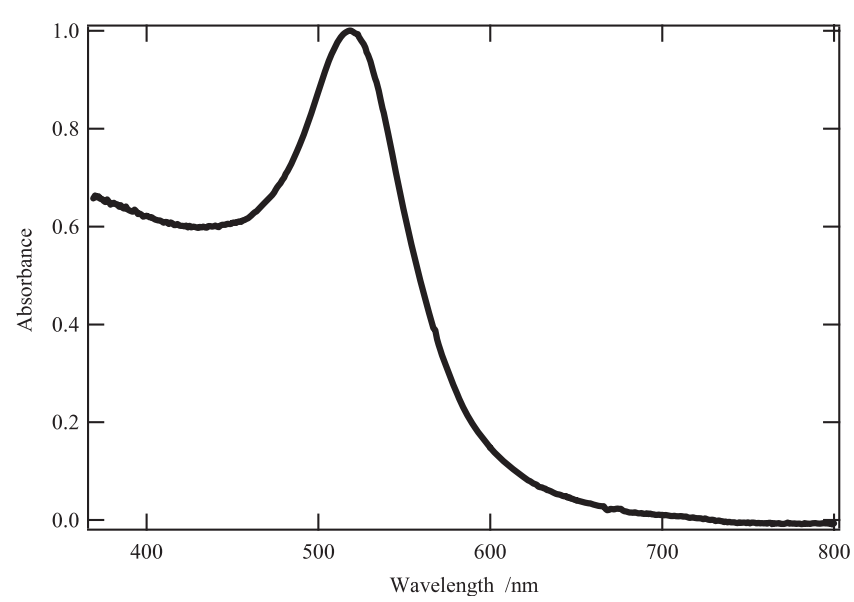

Figure 3. Normalized UV-visible absorbance spectrum of the gold colloidal solution.

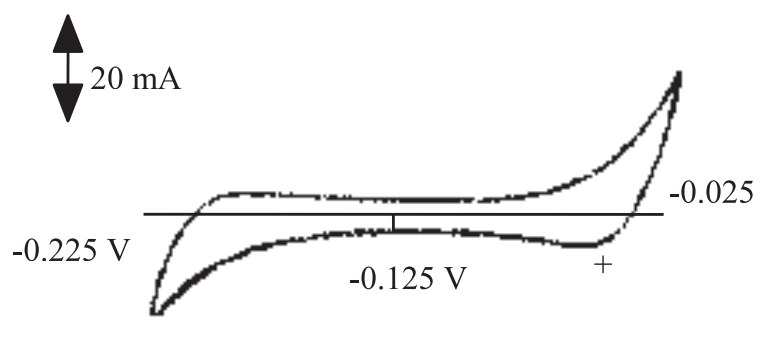

Figure 4. Cyclic voltammogram of the aqueous gold colloids/2-octanone interface. The supporting electrolytes are, respectively, the gold colloids themselves in the aqueous phase and $5 \mathrm{mM}$ THATPBCl in the organic phase. The reference electrodes are $\mathrm{Ag} / \mathrm{AgCl}$ in the aqueous phase and $\mathrm{Ag} / \mathrm{AgTPBCL}$ in the organic phase.

was then passed through a series of lenses and filters to remove any undesirable fundamental residual light and was then collected through a monochromator onto a photomultiplier tube. The signal was then averaged with a boxcar integrator and sent to a microcomputer for further processing.

\section{Results and discussion}

No SSHG signal was observed from the bare water/2-octanone interface. However, in the presence of the colloidal gold solution, a strong SH response from the interface was observed at open circuit arising from the colloidal particles. The light polarization dependence of the SH signal intensity is given by [16]:

$$
I^{\Omega}=\frac{\omega^{2}}{8 \varepsilon_{0} c^{3}} \frac{\sqrt{\varepsilon_{1}^{\Omega}}}{\varepsilon_{1}^{\omega}\left(\varepsilon_{\mathrm{m}}^{\Omega}-\varepsilon_{1}^{\omega} \sin ^{2} \theta_{1}^{\omega}\right)}|\chi|^{2}\left(I^{\omega}\right)^{2}
$$

where $I^{\Omega}$ is the $\mathrm{SH}$ intensity and $I^{\omega}$ is the fundamental intensity; $\varepsilon_{1}^{\omega}$ and $\varepsilon_{1}^{\Omega}$ are the relative dielectric functions of the medium through which the light impinges onto the interface, here the 2-octanone organic phase, at the fundamental and the harmonic frequency, respectively; $\varepsilon_{m}^{\Omega}$ is the relative dielectric function of the interface at the harmonic frequency; and $\theta_{1}^{\omega}$ is the angle 


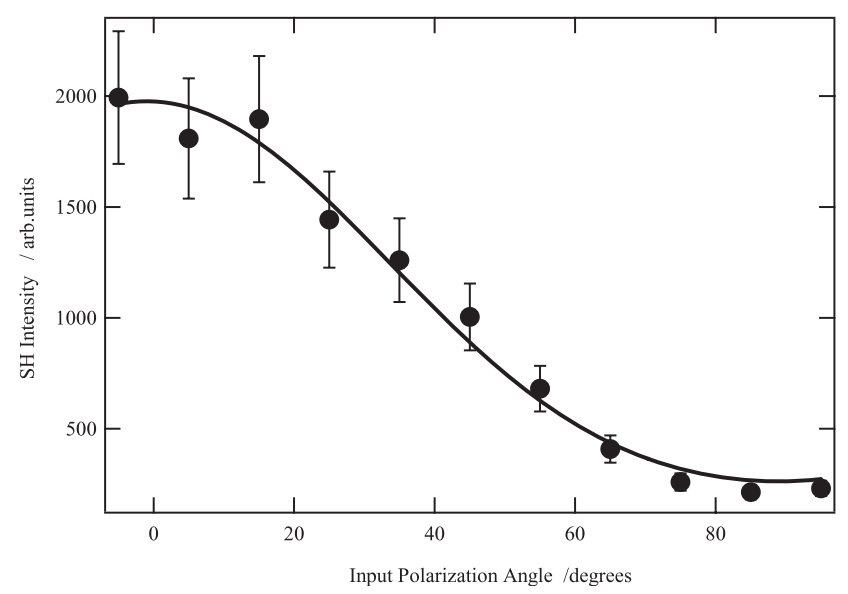

Figure 5. The p-polarized SH intensity as a function of the input polarization angle of the fundamental incoming beam.

of incidence of the fundamental beam. The dielectric function of the interface was taken as the average of the relative dielectric functions of the two bulk phases. Since the absorbance of the colloidal gold solution was low, the imaginary component of the refractive index was disregarded. Thus, all the optical dielectric functions were taken as real quantities and without any dispersion relationships. The quantity $\chi$ in equation (1) is defined as [16]:

$$
\begin{aligned}
\chi=a_{1} \chi_{\mathrm{s}, \mathrm{XZX}}^{(2)} & \sin 2 \gamma \sin \Gamma+\left(a_{2} \chi_{\mathrm{s}, \mathrm{XZX}}^{(2)}+a_{3} \chi_{\mathrm{s}, \mathrm{ZXX}}^{(2)}+a_{4} \chi_{\mathrm{s}, \mathrm{ZZZ}}^{(2)}\right) \cos ^{2} \gamma \cos \Gamma \\
& +a_{5} \chi_{\mathrm{s}, \mathrm{ZXX}}^{(2)} \sin ^{2} \gamma \cos \Gamma
\end{aligned}
$$

where $a_{i}, i=1 \ldots 5$, are coefficients depending on the relative optical dielectric functions and the angle of incidence. The angles of polarization $\gamma$ and $\Gamma$ correspond to the fundamental and the harmonic waves, respectively. The tensor elements $\chi_{\mathrm{S}, \mathrm{XZX}}^{(2)}, \chi_{\mathrm{S}, \mathrm{ZXX}}^{(2)}$ and $\chi_{\mathrm{S}, \mathrm{ZZZ}}^{(2)}$ are the three nonzero independent elements of the interface susceptibility tensor characterizing the nonlinear response of the interface.

With the assumption of an assembly of independent particles at the interface, the signal intensity is directly proportional to $N_{\mathrm{S}}$, the square of the number of particles per unit surface at the interface and the individual hyperpolarizability of the particles. All contributions, intrinsic and extrinsic like the influence of the interface, are embedded into this hyperpolarizability and therefore its magnitude may vary from one interface to another. The surface susceptibility tensor, $\chi_{\mathrm{S}}^{(2)}$, is therefore of the form:

$$
\chi_{\mathrm{S}}^{(2)}=N_{\mathrm{S}}\langle\beta\rangle
$$

where $\beta$ is the hyperpolarizability of a single particle. In equation (3), the brackets indicate an ensemble average. Since the number of particles present at the interface is not known, the absolute magnitude of the hyperpolarizability tensor cannot be deduced. The plots of the SH intensity as a function of the input polarization angle $\gamma$ were first recorded at open circuit. In this regime, the interface is stable and the measured SH intensity is constant. The experiments were carried out as follows. Firstly, the output of the s-polarized light harmonic was recorded. This configuration yielded no signal at all input polarization angles. According to equation (2), it is concluded that the tensor element $\chi_{\mathrm{S}, \mathrm{XZX}}^{(2)}$ vanishes altogether. Secondly, the output of the p-polarized light harmonic was determined and the results are shown in figure 5. From equations (1) and (2), it can be concluded that the susceptibility element $\chi_{\mathrm{S}, \mathrm{ZZZ}}^{(2)}$ dominates over 

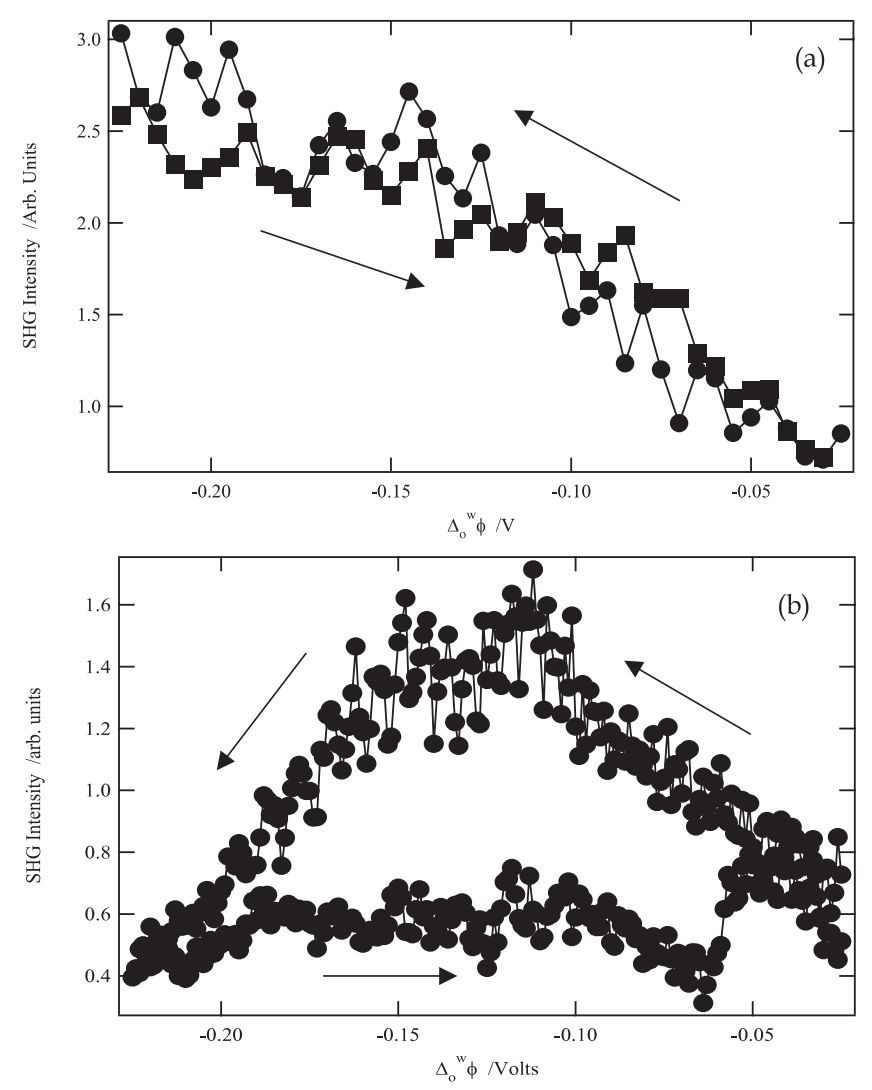

Figure 6. SH signal intensity as a function of the Galvani potential difference for two scan rates: (a) 80 laser pulses averaged every $1 \mathrm{mV}$ and (b) 400 laser pulses averaged every $1 \mathrm{mV}$. The arrow indicates the direction of the potential sweep.

the element $\chi_{\mathrm{S}, \mathrm{ZXX}}^{(2)}$, the ratio of the two elements being $\chi_{\mathrm{S}, \mathrm{ZZZ}}^{(2)} / \chi_{\mathrm{S}, \mathrm{ZXX}}^{(2)}=3$. These results suggest that the interface nonlinearity stems from the liquid/liquid interface itself and not from the particles alone, since breaking of the centrosymmetry is experienced with a translation along the $O Z$ axis, which is the normal to the interface.

Finally, the potential dependence of the SH signal was monitored using electrochemical cell I, and the results are shown in figure 6(a). The Galvani potential difference was swept from -0.025 to $-0.225 \mathrm{~V}$ in steps of $1 \mathrm{mV}$ and the $\mathrm{SH}$ signal was averaged over 80 laser pulses at each potential step. The start and end potentials were chosen according to the cyclic voltammogram shown in figure 4, where the potential window where there is no faradaic current can be seen. The $\mathrm{SH}$ signal intensity exhibits a clear increase as $\Delta_{\mathrm{o}}^{\mathrm{w}} \phi$ is made more negative. Considering that the citrate-stabilized gold nanoparticles carry a net negative charge as a result of the citrate reduction preparation [17, 18], this signal increase results from an increase in the number density of gold particles at the interface, provided that the hyperpolarizability is unchanged. The fact that the SHG signal is monotonous over the potential range explored indicates that the hyperpolarizability is indeed insensitive to the externally applied potential, since the applied field changes sign over this range. Their average interface number density, $N_{\mathrm{s}}$, is in equilibrium with the bulk aqueous number density, $N_{\mathrm{b}}$, through a Boltzmann distribution: 


$$
N_{\mathrm{S}}=N_{\mathrm{b}} \exp \left(\frac{z b F}{R T} \Delta_{\mathrm{o}}^{\mathrm{w}} \phi\right)
$$

where $\Delta_{\mathrm{o}}^{\mathrm{w}} \phi$ is the Galvani potential difference across the liquid/liquid junction, $z$ is the charge retained by the particle, and $b$ is the fraction of the full potential drop $\Delta_{\mathrm{o}}^{\mathrm{w}} \phi$ present between the bulk aqueous phase and the plane of adsorption of the particles at the interface. Equation (4) is only valid at low coverage where the adsorbed layer can be regarded as ideal, at potentials not too negative and for slow scan rates, where the approximation of the thermodynamic equilibrium is still valid and aggregation is negligible. At more negative potentials, a more refined isotherm should be used. Furthermore, aggregation would probably complicate the analysis. The adsorption of charged species at polarized liquid/liquid interfaces has already been observed and the value obtained for $b$ has always been found to be much smaller than unity. For example, for the dye methyl orange, a value of 0.22 has been found, indicating that the plane of adsorption is located significantly into the aqueous phase [19]. From equations (1) to (4), a value of 0.14 was found for the product $z b$, i.e between the charge retained by the colloid particle and the potential drop in the aqueous phase between the bulk solution and the adsorption plane. It appears, therefore, that the gold particles either do not retain a very large effective electrostatic charge or do not sense the micropotential at the adsorption plane. The latter case can result from the large size of the nanoparticles studied, of approximately $20 \mathrm{~nm}$, and the presence of free electrons in the metal cores, which will strongly distort the potential distribution between the two phases.

The results shown in figure 6(a), recorded within $1600 \mathrm{~s}$ (as calculated from the repetition rate of the laser), the number of potential steps and the number of laser pulses averaged demonstrate that the adsorption process is fully reversible. However, for the results obtained at much slower scan rates (400 laser pulses per potential step; see figure 6(b)), the potentialdependent adsorption process is no longer reversible and aggregation of the particles at the interface is no longer negligible, as observed through the loss of the SH signal. This loss occurs because, upon aggregation, the SP resonance at $532 \mathrm{~nm}$ (the harmonic wavelength) decreases considerably [20]. Clearly, in this case the assumption of independent particles is no longer valid at high surface number densities where particles can interact. It has been reported that, in such a case, overlapping of the electronic wavefunctions and the subsequent electron density build-up between the particles yields a sharp increase in the nonlinear susceptibility tensor magnitude [4, 21]. This effect was observed because organically functionalized silver particles were used. The increase in the nonlinear susceptibility tensor occurred for inter-particle distances as short as $1 \mathrm{~nm}$, and thus the alkylthiol passivating layer prevented coalescence. In the experiments reported here, the particles are not functionalized and therefore the regime of close proximity of the particles without aggregation is never obtained. By contrast, at high surface coverage, the particles coalesce into larger ensembles and the SH intensity drops as a result of the loss of the surface plasmon resonance enhancement. This decrease arises from both a red shift of the surface plasmon resonance and the consequent loss in coupling efficiency of the SH field to the surface plasmon for larger particles [20].

In order to follow the time evolution of the SH signal during coalescence, potential step experiments were also performed. The cell was first maintained for a couple of minutes at open circuit during which time the fine adjustments of the optics of the system such as focusing and alignment were carried out. An initial Galvani potential difference of $-0.025 \mathrm{~V}$ was applied, since gold particles are not irreversibly adsorbed at this potential due to the low surface coverage decreasing aggregation significantly. The Galvani potential difference was then stepped to $-0.125 \mathrm{~V}$ (figure 7 ) or $-0.225 \mathrm{~V}$ (figure 8). A very sharp rise in the SH signal to its maximum value was observed immediately following the potential step to $\Delta_{\mathrm{o}}^{\mathrm{w}} \phi=-0.125 \mathrm{~V}$. 


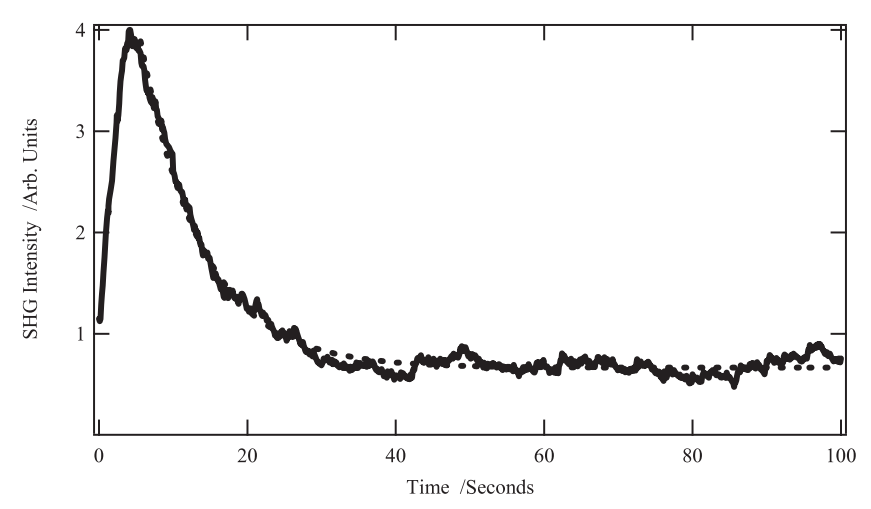

Figure 7. SH signal intensity as a function of time for a Galvani potential difference of $-0.125 \mathrm{~V}$.

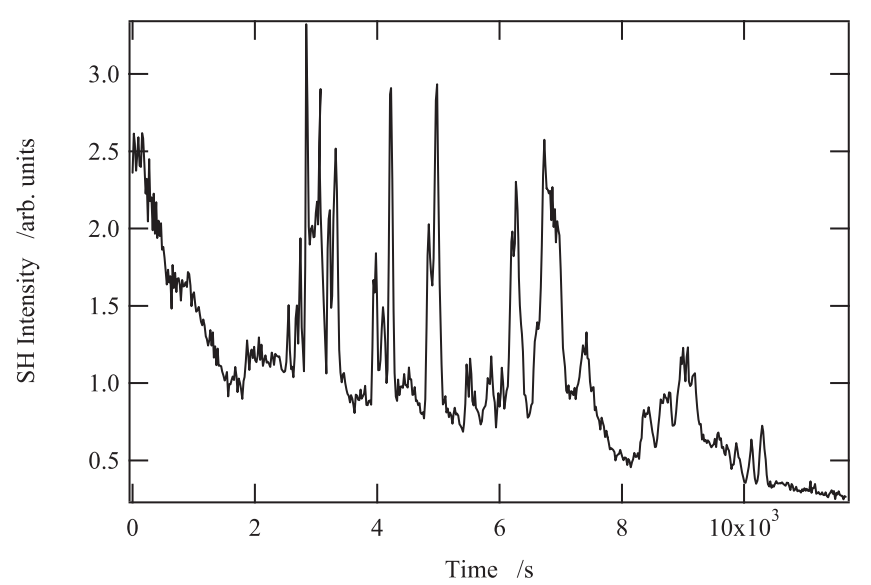

Figure 8. SH signal intensity as a function of time for a Galvani potential difference of $-0.225 \mathrm{~V}$.

But this part of the curve is not observed in figure 8. The sharp rise in the SH signal is attributed to the adsorption of the particles at the interface in the regime where equations (1) to (4) are valid. The signal then decayed as expected from the coalescence process. The maximum observed is due to the effects of competition between particle adsorption, surface diffusion and aggregation processes, eventually leading to the final loss of the SHG signal. The citrate-gold colloid carries a large total negative charge and hence it would be expected that adsorption and aggregation are strongly potential dependent. Thus, for $\Delta_{\mathrm{o}}^{\mathrm{w}} \phi=-0.125 \mathrm{~V}$, the rate of adsorption is overrun by the rate of aggregation within seconds, as is seen from the single exponential decay in figure 7 , with a half-time decay time constant of approximately $10 \mathrm{~s}$. In contrast, for $\Delta_{\mathrm{o}}^{\mathrm{w}} \phi=-0.225 \mathrm{~V}$, the rate of adsorption is much faster and partially compensates the rate of surface diffusion and aggregation, leading to the loss of the SHG signal. Hence the apparent decay of the SHG signal is slower, in this case approximately 1300 s. At long timescales, strong fluctuations much larger than the laser fluctuations were observed, with the SH signal going back its maximum value. These recurrences result from the diffusion of large islands of coalesced particles in and out from the laser spot at the interface, leaving island-free regions under the laser spot. In these regions, the remaining non-coalesced smaller particles again yield a strong signal. It is difficult at present to perform a statistical analysis of these fluctuations, but work along the lines reported by Zhao et al could well be 
envisaged [22]. Such an analysis would be desirable for disentangling any other possible effects such as instabilities [23]. It is interesting to note here that the timescale of the loss of the correlation for these fluctuations, as deduced from the decay of the correlation function $g^{(2)}(\tau)=\langle I(t+\tau) I(t)\rangle /\langle I(t)\rangle^{2}$, is rather long (approximately $300 \mathrm{~s}$ ). Such a characteristic time cannot be attributed to the surface diffusion of single particles but rather to large domains with large areas, in accordance with the proposed mechanism of loss of the SH signal.

\section{Conclusions}

Colloidal gold particles have been observed at the polarized water/2-octanone interface using surface second harmonic generation, and the potential dependence behaviour of the SH signal is reported. The potential dependence of the adsorption process is demonstrated, and it is shown that reversibility of the process may be achieved with sufficiently fast scan rates. Indeed, at too low scan rates the surface diffusion and aggregation of the particles compete efficiently with adsorption. This is further demonstrated with the time evolution of the SHG signal. The rate of adsorption is potential dependent and therefore the rate of loss of the SHG signal due to aggregation is potential dependent. Surface diffusion of the aggregated domains under the laser spot is also clearly observed through the SH signal fluctuations. It can be concluded that a time window for reversible behaviour of this system may be found, but it is rather short.

\section{References}

[1] Kreibig U and Vollmer M 1995 Springer Series in Material Science 25 (Berlin: Springer)

[2] Hayat M A (ed) 1989 Colloidal gold Principles, Methods and Applications (New York: Academic)

[3] Cheng Y and Schiffrin D 1996 J. Chem. Soc. Faraday Trans. 923865

[4] Collier C P, Saykally R J, Shiang J J, Henrichs S E and Heath J R 1997 Science 2771978

[5] Remacle F, Collier C P, Heath J R and Levine R D 1998 Chem. Phys. Lett. 291453

[6] Lahtinen R, Fermín D J, Kontturi K and Girault H H 2000 J. Electroanal. Chem. 48381

[7] Eisenthal K B 1996 Chem. Rev. 961343

[8] Brevet P F and Girault H H 1996 Liquid-Liquid Interfaces. Theory and Methods (Boca Raton, FL: CRC Press)

[9] Antoine R, Brevet P F, Girault H H, Bethell D and Schiffrin D J 1997 Chem. Commun. 1901

[10] Nappa J, Revillod G, Russier-Antoine I, Benichou E, Jonin C and Brevet P F 2005 Phys. Rev. B 71165407

[11] Nappa J, Russier-Antoine I, Benichou E, Jonin Ch and Brevet P F 2005 Chem. Phys. Lett. 415246

[12] Agarwal G S and Jha S S 1982 Solid State Commun. 41499

[13] Dadap J I, Shan J, Eisenthal K B and Heinz T F 1999 Phys. Rev. Lett. 834045

[14] Clarke D J, Schiffrin D J and Wiles M C 1989 Electrochim. Acta 34767

[15] Turkevich J, Stevenson P C and Hillier J 1951 Discuss. Faraday Soc. 1155

[16] Brevet P F 2001 Nonlinear optics at liquid/liquid interfaces Liquid Interfaces in Chemical, Biological and Pharmaceutical Applications (Surface Science Series vol 95) ed A G Volkov (New York: Dekker)

[17] Kuiper A C 1933 J. Am. Chem. Soc. 551722

[18] Freeman R G, Hommer M B, Grabar K C, Jackson M A and Natan M J 1996 J. Phys. Chem. 100718

[19] Piron A, Brevet P F and Girault H H 2000 J. Electroanal. Chem 48329

[20] Galletto P, Brevet P F, Girault H H, Antoine R and Broyer M 1999 J. Phys. Chem. B 1038706

[21] Shiang J J, Heath J R, Collier C P and Saykally R J 1998 J. Phys. Chem. B 1023425

[22] Zhao X, Goh M C, Subrahmanyan S and Eisenthal K B 1990 J. Phys. Chem. 943370

[23] Kovalchuk N M and Vollhardt D 2005 J. Phys. Chem. B 10922868 